\title{
A datalogger to identify vocalizing dolphins
}

\author{
Peter L. Tyack and Cheri A. Recchia \\ Department of Biology, Woods Hole Oceanographic Institution, Woods Hole, Massachusetts 02543
}

(Received 3 January 1991; accepted for publication 7 May 1991)

\begin{abstract}
A datalogger was developed to identify vocalizing dolphins within socially interacting captive groups. Every $50 \mathrm{~ms}$ the logger stores data on the level and frequency of detected sound.

Dataloggers are temporarily attached to dolphins by suction cups for data collection sessions lasting up to $45 \mathrm{~min}$. Later, computer analysis of data from the dataloggers reveals which dolphin produced each vocalization recorded during the session. Results from use of dataloggers with two captive bottlenose dolphins (Tursiops truncatus) at the New England Aquarium in Boston, MA are presented. The possible use of dataloggers with wild dolphins is discussed.
\end{abstract}

PACS numbers: 43.80.Jz, 43.80.Nd, 43.80.Lb

\section{INTRODUCTION}

Bottlenose dolphins, Tursiops truncatus, produce a wide variety of vocalizations including both broad- and narrowband sounds. Of these, the most intensively studied sounds are the broadband pulsed signals used for echolocation, and the narrow-band frequency-modulated calls termed whistles or squeals (Herman and Tavolga, 1980; Watkins and Wartzok, 1985). Studies of whistle function are best accomplished through identification of vocalizing animals within socially interacting groups. However, the inability of humans to localize underwater sound sources makes this problematic.

Tyack (1985) developed a small acoustic telemetry device, the vocalight, to identify vocalizing dolphins. Vocalights are temporarily attached to the animals' heads with suction cups, and contain light-emitting diodes which illuminate upon detection of sounds such as dolphin whistles. The vocalight was used to study the vocal behavior of two captive bottlenose dolphins at the Sealand Aquarium in Brewster, MA. However, it is impractical for use with more than two or three animals because it is difficult to observe all vocalights simultaneously and continuously. Even with only two dolphins, it is not possible to identify which individual produced every whistle. With larger groups of dolphins, the proportion of identifiable whistles will be further decreased. Many analysis techniques require unbroken sequences of whistles identified to producer.

We describe here a datalogger developed by Tyack to identify vocalizing individuals within a group of freely interacting captive dolphins. The datalogger stores information about sounds it detects for later computer analysis. This method avoids the problems described above and might be applicable to a study of wild dolphins under certain conditions. We present results from successful use of dataloggers with two bottlenose dolphins at the New England Aquarium in Boston, MA.

\section{MATERIALS AND METHODS}

\section{A. Design of datalogger}

The datalogger consists of a Tattletale model IV computer (Onset Computer Corporation, North Falmouth,
MA) with $160 \mathrm{~kb}$ RAM, and a signal conditioning circuit (designed by R. Koehler and P. Tyack of the Woods Hole Oceanographic Institution) that modifies the signal received from a hydrophone and preamplifier. The hydrophone signal is amplified, filtered, and split into two channels: one for determining the level of the signal and the other for determining frequency. Level is determined using an rms to dc converter. Frequency is determined by clipping the signal to a 5-V square wave, dividing zero crossings by two (because whistles range up to $20 \mathrm{kHz}$, but the Tattletale PERIOD command returns incorrect values for frequencies over 10 $\mathrm{kHz}$ ), and timing zero crossings in groups of ten to a precision of $0.8 \mu \mathrm{s}$. The Tattletale greatly simplifies the timing of datalogging, using a language similar to BASIC with built-in sampling at intervals of multiples of $10 \mathrm{~ms}$. In our application, we sample 20 times per second (50-ms interval), and record the time and level of all signals and the frequency of signals above a programmed threshold. The unit is powered by a standard $9-\mathrm{V}$ battery.

The datalogger stores data in 1, 2, or 4 byte units of memory. The analog to digital converter is a 10 -bit converter. If we store all 10 bits, we must use 2 bytes ( 16 bits) of memory. This leaves 6 bits free in each 2-byte number stored. We store the low five bits of a counter that increments the number of 10-ms timing intervals. This allows us to detect whether the logger developed timing errors while logging data. The low five bits of this counter cover an interval of 320 $\mathrm{ms}$, much longer than any timeout we have detected.

The datalogger is housed in a waterproof polycarbonate (Lexan) case sealed with a metal endplate and a flat neoprene gasket (Fig. 1). The endplate is fitted with a watertight input/output (I/O) port used to link the datalogger to a computer. The computer is used to program the unit, to activate the unit at the beginning of a data collection session, and to download the digital data after a session. The datalogger housing also contains a liquid crystal display (LCD), which displays either timing data or a cumulative total of datalogger memory used during a data collection session. We have found the LCD to be extremely useful for monitoring memory usage and detecting malfunctions during data collection. 


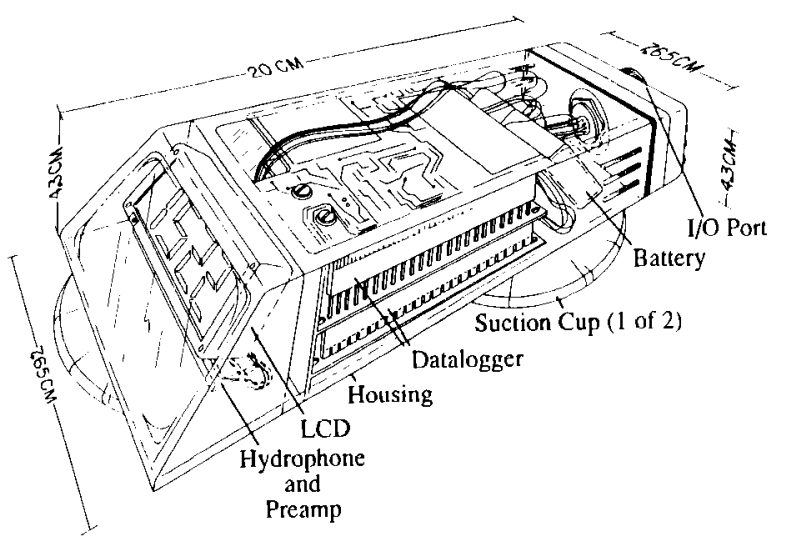

FIG. 1. Diagram of datalogger in waterproof Lexan housing. The two urethane suction cups hold the unit on the animal. The package weighs approximately $0.5 \mathrm{~kg}$ in air, and is neutrally buoyant in seawater.

\section{B. Attachment}

We tested two different methods of attaching the dataloggers to the dolphins. Both methods were developed in close collaboration with $\mathrm{K}$. Krieger and the marine mammal training staff of the New England Aquarium. The first method involved a custom-made backpack and harness, and the second consisted of two suction cups mounted on the bottom of each datalogger. The forward suction cup on each logger was mounted on the housing directly under the piezoelectric crystal, forming a contact hydrophone. Both attachment methods were evaluated on the basis of ease and security of attachment, amount of training required for the dolphins to wear the dataloggers, and effects of wearing the dataloggers on the dolphins' behavior. Effects of attachment method on the quality of data obtained were examined by comparing the output from suction cup and harness dataloggers worn simultaneously by the same dolphin.

\section{Procedure}

Immediately prior to a datalogger session, the dataloggers were linked to a computer, loaded with the data collection program, and activated. After activation, the units were in a "pause" mode awaiting a 5-V start signal. The units were then linked to a SMPTE time code generator, which sent the start signal to all dataloggers simultaneously. Thus synchronized, the dataloggers were ready for the data collection session. The time code generator also time-stamped a video recording of the session, synchronized to the datalogger start signal. A video camera mounted over the dolphin pool provided a visual record of the behavior and positions of the animals during the datalogger session. Hydrophones, mounted inside the pool and connected to two hi-fi audio channels of the VCR, provided an analog record of vocalizations for later spectrographic analysis. A microphone was connected to a normal audio channel for observer comments.

Once activated and synchronized, the dataloggers were placed on the dolphins by the trainers. We noted which datalogger was placed on which dolphin. The dolphins were then allowed to swim and interact freely, while an observer made detailed behavioral observations and periodically checked the LCD of each datalogger to monitor data storage. Ideally, the session continued until an LCD indicated that a datalogger's memory was full, approximately $45 \mathrm{~min}$. The dolphins were then recalled, the dataloggers removed, and the data downloaded into the computer.

We analyzed the logger data in our laboratory. The videotape was reviewed, and times at which whistles occurred during the datalogger session were noted. Each such time was then entered into a computer program that determines which datalogger recorded the loudest signal at that particular time. The datalogger with the loudest signal is assumed to have been on the whistling dolphin. This process was repeated for all times whistles occurred during the datalogger session to determine which dolphin made each whistle.

Currently we analyze the acoustic structure of each whistle by visual inspection of sound spectrographs produced by a Kay DSP Sona-Graph (model 5500 ). Linking each whistle to logger data is easiest with a graphic plot of signal level from each logger at the time of the whistle in question and of the frequency contour from the logger with the loudest signal. The contour plot helps the observer to assure that a loud level was caused by the whistle and not by other sources of noise, such as the dolphin leaping out of the water or rubbing the logger against the wall of the pool. These other sources of noise can be confirmed by viewing the videotape. As we gain experience with potential sources of error, we hope to automate the entire process of whistle identification and analysis. The datalogger technique is well suited to such automation.

\section{RESULTS}

The dataloggers were tested with captive bottlenose dolphins at the New England Aquarium. The facility housed three dolphins during the data collection sessions reported here: two 11-year-old males and one 8-year-old female (ages are approximate). The female, Tapeko, had been in captivity for less than 2 years and had not yet learned to wear a datalogger. The males, Sandy and Rainbow, had already been trained to wear eye cups for echolocation demonstrations. It took approximately 1 month of additional training for the animals to wear the suction cup dataloggers. Both Sandy and Rainbow seemed to habituate fairly quickly to wearing the suction cup dataloggers, usually swimming and interacting apparently normally during datalogger sessions. The dolphins appeared to prefer wearing the loggers either just caudal to the blowhole on the midline, or lateral to the dorsal fin.

Neither Sandy nor Rainbow had worn a harness before, and it took considerable time and training before they would wear the harness dataloggers. Over a period of several months, the dolphins were trained to wear a single girth, then two girths, then the complete harness. However, despite extensive training, neither dolphin seemed to habituate completely to wearing the harness. During datalogger sessions, the dolphin's behavior was clearly affected by wearing the harnesses: The animals oriented much more toward the trainers and tended to stay near the training platform, rather than interacting with each other. 
Further, a comparison of data logged by harness and suction cup dataloggers worn simultaneously by Rainbow showed a higher noise component and lower signal levels from the harness datalogger (Fig. 2). During the session, two temporally overlapping whistles were recorded with a hydrophone in the pool, the first faint, the second loud. As the logger output shows, both dataloggers recorded the second whistle only, suggesting that the first whistle was not produced by Rainbow, but the second one was. The higher peaks in the uppermost right plot depict the higher signal levels received by the suction cup logger. The harness logger recorded lower signal levels despite being physically closer to Rainbow's head. The harness logger data also typically contained a significantly higher noise component. Subsequent datalogger sessions were conducted using only the suction cup dataloggers.

In Fig. 3, data are presented from a datalogger session in which both Rainbow and Sandy were wearing suction cup dataloggers. These data were collected during training sessions where the dolphins were in adjacent pools. Each pool is approximately $8 \mathrm{~m}$ long $\times 6 \mathrm{~m}$ wide $\times 2 \mathrm{~m}$ deep. Tapeko was in the same pool as Sandy, and was not wearing a datalogger. Both dataloggers appear to have detected this whistle series, but the level was much higher on Sandy's logger than on Rainbow's. The lower signal levels recorded by Rainbow's logger suggest that Rainbow did not produce that whistle series. However, since Tapeko was not wearing a logger, it is not possible to determine whether the whistle series shown was produced by Sandy or Tapeko. The videotape shows that this whistle series was produced while Sandy and Tapeko were interacting and were physically very close.

\section{DISCUSSION}

Although the harness held the datalogger in a good position, just caudal to the blowhole and near the source of whis-
VIDEOTAPE

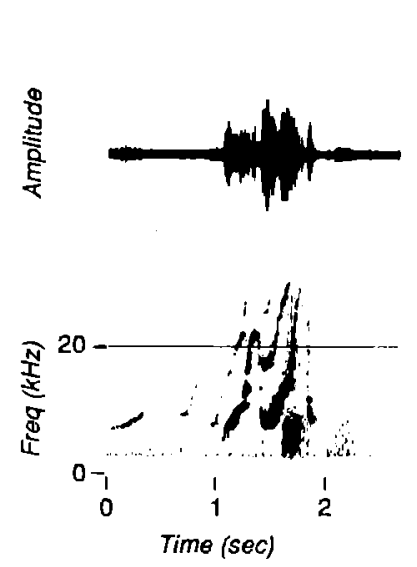

DATALOGgERS

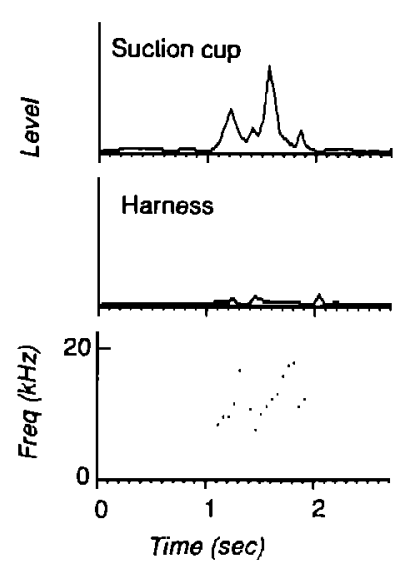

FIG. 2. Comparison of output from two dataloggers worn simultaneously by Rainbow. On the left are the waveform (upper) and spectrograph (lower) generated by spectrographic analysis of two temporally overlapping whistles recorded with a hydrophone in the pool (analyzing filter bandwidth was $469 \mathrm{~Hz}$ ). On the right are the levels of the same signal as recorded by a suction cup logger (uppermost plot) and a harness logger (middle plot). The lowermost right plot is the frequency contour of the signal as recorded by the suction cup logger.
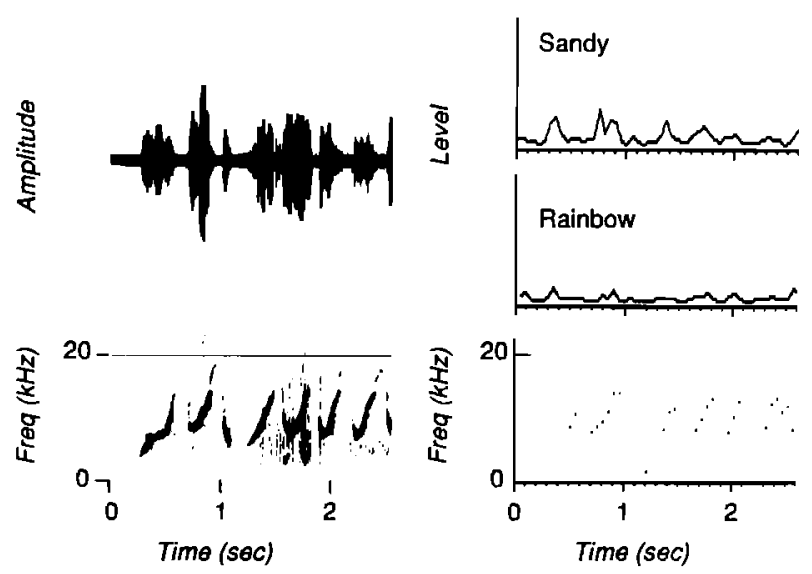

FIG. 3. Comparison of output from dataloggers worn simultaneously by two dolphins. On the left are the waveform (upper) and spectrograph (lower) generated by spectrographic analysis of a whistle series recorded with a hydrophone in the tank (analyzing filter bandwidth was $469 \mathrm{~Hz}$ ). On the right are the levels of the same signal as recorded by a logger on Sandy (uppermost plot) and on Rainbow (middle plot). The lowermost right plot is the frequency contour of the signal as recorded by Sandy's logger.

tles, the harness loggers recorded much lower levels than those attached with suction cups. This difference appears to have several causes: (1) Hydrodynamic drag generated by the harness and backpack increased noise on harness loggers; (2) harness loggers were more loosely attached and could bang against the dolphin's back; and (3) the suction cup mounted directly under the hydrophone provided excellent acoustic coupling for the suction cup loggers. The acoustic advantage of the suction cup attachment was very striking. Even when the suction cup logger was attached lateral to the dorsal fin, it recorded much higher levels than a harness logger attached just caudal to the blowhole.

The datalogger described here clearly will be useful for study of individual vocal repertoires and social functions of dolphin vocalizations. Description of the responses of "listening" dolphins to a whistling individual requires identification of the vocalizing animal. Use of dataloggers permits such identification without limiting the movements or interactions of the study animals. Since a group of captive animals can be studied as a whole, breaking the group up into one or a few animals to ensure identification of the vocalizing animal is unnecessary. Thus observation of more complex social situations is possible.

Use of suction cups for attachment of the dataloggers to the animals may allow use with wild dolphins under certain circumstances. For example, the animals could be captured briefly for attachment of the dataloggers, then released into a net corral. Since the dolphins can dislodge the dataloggers simply by arching their backs, recapturing the animals to remove the units would be unnecessary. We have attached vocalights and suction cup hydrophones to wild dolphins temporarily restrained inside net corrals near Sarasota, FL and observed little resultant change in behavior (Sayigh et al., 1990). Our work at the New England Aquarium suggests that in order to identify with certainty which dolphin in 
a group produces a sound, each animal in the pool must wear a datalogger. However, in the wild, animals are often separated by larger ranges, so it may be possible to work with single focal animals wearing loggers.

\section{ACKNOWLEDGMENTS}

We thank Kathy Krieger and the other marine mammal trainers at the New England Aquarium in Boston, MA and the training staff of the Seven Seas pavilion of the Brookfield Zoo in Brookfield, IL for their help in solving the datalogger attachment problem, for training the dolphins to wear the dataloggers, and for cheerfully tolerating our often inconvenient requests. Thanks also to Dan Smith for programming assistance, and to William Watkins and Kurt Fristrup for reviewing the manuscript. This work was supported by ONR Grant No. N00014-87-K-0236. This is Contribution No. 7586 from the Woods Hole Oceanographic Institution.

Herman, L. M., and Tavolga, W. N. (1980). "The communication systems of cetaceans," in Cetacean Behavior: Mechanisms and Functions, edited by L. M. Herman (Wiley, New York), Chap. 4, pp. 149-209.

Sayigh, L. S., Tyack, P. L., Wells, R. S., and Scott, M. D. (1990). "Signature whistles of free-ranging bottlenose dolphins Tursiops truncatus: stability and mother-offspring comparisons," Behav. Ecol. Sociobiol. 26, 247-260.

Tyack, P. (1985). "An optical telemetry device to identify which dolphin produces a sound," J. Acoust. Soc. Am. 78, 1892-1895.

Watkins, W. A., and Wartzok, D. (1985). "Sensory biophysics of marine mammals," Mar. Mamm. Sci. 1, 219-260. 